\title{
Development of a radio frequency quadrupole linac implemented with the equipartitioning beam dynamics scheme
}

\author{
Yasuhiro Kondo* and Takatoshi Morishita \\ Japan Atomic Energy Agency (JAEA), Tokai, Naka, Ibaraki 319-1195, Japan \\ Robert A. Jameson \\ Institüt Angewandte Physik, Goethe-Universität Frankfurt, Frankfurt am Main D60438, Germany
}

(Received 9 October 2019; published 23 December 2019)

\begin{abstract}
Radio frequency quadrupole linac (RFQ) is the key component which realized modern high-current proton linacs, however, many RFQs are designed based on very conventional design schemes. We developed 3-MeV 50-mA H${ }^{-}$(negative hydrogen) RFQ based on a beam space-charge physics concept. The equipartitioning scheme, which is widely used in the high intensity linac design, is implemented into the RFQ design. Design performances of $99.1 \%$ transmission, $0.24 \pi \mathrm{mm}$ mrad transverse normalized rms emittance, and $0.11 \pi \mathrm{MeV}$ deg for longitudinal direction are achieved with a vane length of $3 \mathrm{~m}$ and the final energy of $3 \mathrm{MeV}$. An RFQ employing this design concept was fabricated, and the design performance was confirmed by beam commissioning. In this paper, the design and result of the beam commissioning of this fully equipartitioned RFQ are described.
\end{abstract}

DOI: 10.1103/PhysRevAccelBeams.22.120101

\section{INTRODUCTION}

The radio frequency quadrupole (RFQ) is the key component which realized modern high-current proton linacs. However, even now, many RFQs are designed based on very conventional design schemes. Although the transverse and longitudinal beam dynamics are coupled via the space-charge force of a high beam current, conventional methods design the transverse and longitudinal dynamics independently. The method proposed by Los Alamos National Laboratory [1] is one of these conventional schemes. In this method, an RFQ is divided into four sections: a radial matching section (RMS), a shaper (SP), a gentle buncher (GB), and an accelerator (ACC). In the GB, a scheme for the adiabatic bunching is implemented, but it focused on only the longitudinal dynamics. Additionally, by the fabrication considerations, intervane voltage $V$ and average bore radius $r_{0}$ are kept constant except for the RMS; this means the transverse focusing strength $B$ is kept constant. Therefore, $B$ remains strong even in the ACC, where the space-charge force is relatively weak and the transverse focusing force needs not to be strong. This causes insufficient longitudinal force and ineffective acceleration efficiency. Furthermore, a weaker transverse

\footnotetext{
*yasuhiro.kondo@j-parc.jp
}

Published by the American Physical Society under the terms of the Creative Commons Attribution 4.0 International license. Further distribution of this work must maintain attribution to the author(s) and the published article's title, journal citation, and DOI. focusing and stronger longitudinal focusing at the RFQ exit make it easier to match to the following linac such as drift tube linac. In these days, to increase the acceleration efficiency, some RFQs are designed to reduce $B$ (increase $r_{0}$ ) and make $V$ higher with progress of acceleration to obtain more acceleration efficiency [2,3]. However, the beam dynamics itself is still not based on directly controlled and self-consistent space-charge physics.

To improve this situation, one of the authors (R.A. Jameson) proposed to use the equipartitioning beamdynamics scheme in the RFQ design [4]. With this method, the beam dynamics is purely based on the correct beam physics with space charge, achieved with flexible choices of the main parameters. The transverse and longitudinal forces are naturally balanced by the equipartitioning scheme and the efficient acceleration can be achieved. So, we call this RFQ design method as "beam oriented RFQ design." We implemented this method into a new RFQ. This RFQ is a spare of the J-PARC (Japan proton accelerator research complex) $\mathrm{H}^{-}$(negative hydrogen) RFQ, which called RFQ III [5]. In Table I, the specifications of the J-PARC RFQ are listed.

In the design of RFQ III, the conventional constant- $r_{0}$ and - $V$ design was adopted, and the equipartitioning was implemented only in the GB [6]. However, we accumulated a lot of experience through the fabrication of RFQ III and established an effective tuning method. Here, we considered the prospects for realizing an equipartitioned RFQ, and finally we decided to adopted the fully equipartitioning design.

In this paper, the beam-dynamics design and the beam commissioning result of the fully equipartitioned RFQ 
TABLE I. Requirements for J-PARC RFQ.

\begin{tabular}{lc}
\hline \hline Beam species & $\mathrm{H}^{-}$ \\
Resonant frequency & $324 \mathrm{MHz}$ \\
Injection energy & $50 \mathrm{keV}$ \\
Extraction energy & $3 \mathrm{MeV}$ \\
Peak beam current & $50 \mathrm{~mA}$ \\
Transverse normalized rms emittance & $<0.25 \pi \mathrm{mm} \mathrm{mrad}$ \\
Repetition rate & $50 \mathrm{~Hz}$ \\
rf pulse length & $600 \mu \mathrm{s}$ \\
rf duty factor & $3 \%$ \\
\hline \hline
\end{tabular}

(epRFQ) are described. The detail of the beam oriented RFQ design scheme is shown in Sec. II. In Sec. III, the particle simulation results are presented. The results of the beam commissioning are shown in Sec. IV. Finally, in Sec. V, we summarize the paper.

\section{BEAM ORIENTED RFQ DESIGN}

In the equipartitioning (EP) scheme, beam internal energy balance is defined with the emittance $\varepsilon$ and the phase advance $\sigma$ as being equipartitioned to the longitudinal and transverse directions when

$$
\frac{\varepsilon_{l n} \sigma_{l}}{\varepsilon_{t n} \sigma_{t}}=1,
$$

where $\varepsilon_{l n}$ and $\varepsilon_{t n}$ are the longitudinal and transverse normalized rms emittances, and $\sigma_{l}$ and $\sigma_{t}$ are longitudinal and transverse phase advances defined over the same unit length. If this condition is satisfied, there is no free energy to drive the parametric resonances in high-current linacs [7].

In the beam dynamics of the RFQ, the beam is required to be a matched beam in a periodic focusing system. The matched beam is described using the smooth approximation as

$$
\begin{gathered}
\varepsilon_{t n}=\frac{r^{2} \sigma_{t} \gamma}{L}, \\
\varepsilon_{l n}=\frac{(\gamma b)^{2} \sigma_{l} \gamma}{L},
\end{gathered}
$$

where $r$ and $b$ are the transverse and longitudinal beam radii, $L$ is a suitable unit length [8] the same for both dimensions, and $\gamma$ is the relativistic gamma. Furthermore, Eqs. (2) and (3) are described using the relation between the phase advances with a beam current $I\left(\sigma_{t}, \sigma_{l}\right)$ and without it $\left(\sigma_{0}^{t}, \sigma_{0}^{l}\right)$ as

$$
\begin{aligned}
& \varepsilon_{t n}^{2}=\frac{r^{4} \gamma^{2}}{L^{2}}\left\{\sigma_{0}^{t 2}-\frac{I L^{3} k(1-f)}{r^{2}(\gamma b) \gamma^{2}}\right\}, \\
& \varepsilon_{l n}^{2}=\frac{(\gamma b)^{4} \gamma^{2}}{L^{2}}\left\{\sigma_{0}^{l 2}-\frac{2 I L^{3} k f}{r^{2}(\gamma b) \gamma^{2}}\right\} .
\end{aligned}
$$

The constant $k=\frac{3}{8 \pi} \frac{Z_{0} q \times 10^{-6}}{m_{0} c^{2}}$ is written with impedance of vacuum $Z_{0}$, the charge and the rest mass of the particle $q$ and $m_{0}$, the velocity of light $c$, and the ellipsoid form factor $f$ [9]. The $\sigma_{0}^{t}$ and $\sigma_{0}^{l}$ are defined by the structural parameters of the focusing system as

$$
\begin{gathered}
\sigma_{0}^{t 2}=\frac{B}{8 \pi^{2}}+\Delta_{r f}, \\
\sigma_{0}^{l 2}=2 \Delta_{r f} .
\end{gathered}
$$

The focusing factor $B$ is

$$
B=\frac{q \lambda^{2} V}{m_{0} c^{2} r_{0}^{2}},
$$

and the rf refocusing factor $\Delta_{r f}$ is

$$
\Delta_{r f}=\frac{\pi^{2} q V A \sin \phi_{s}}{2 m_{0} c^{2} \beta^{2} \gamma^{3}}
$$

where $\phi_{s}$ is the synchronous phase angle and $\beta$ is the relativistic beta [1]. The acceleration parameter $A$ is described with multipole components of the external field as $A=A_{10}+A_{30}$. The $A_{10}$ and $A_{30}$ are parametrized with the geometrical parameters of the vane tip, that is, the vane modulation factor $m$, the aperture radius $a$ described at the vane tip minimum, and the cell length $l_{c}[10]$. From these equations, the EP condition Eq. (1) can be calculated, and varying the parameters of the RFQ by keeping the EP condition is the essence of beam oriented RFQ design.

For the beam-dynamics design of epRFQ, LINACSrfqDES [4,11] was used. The most essential feature of LINACSrfqDES is the beam-based design, i.e., the ability to design RFQs including the space-charge physics. In LINACSrfqDES, the distinction between the GB and ACC no longer exists, instead, the same rule is applied to the whole RFQ after the exit of the SP, where the achievement of equipartitioning is first required. The independent variables in LINACSrfqDES are $V, m, a$, and $\phi_{s}$, which must be specified by rules as functions of cell number or beta or position, etc. They also may be used to solve for secondary variables such as the EP condition or $B$, etc. along the RFQ. The $a$ may be varied as a function of the particle velocity $\beta$. The $V$ may also be varied; in this design it is increased so that the ratio of the surface electric field to the empirical Kilpatrick limit $E_{k}$ [12] is kept constant. The rule used in this design for the synchronous phase $\phi_{s}$ is

$$
\begin{gathered}
\phi_{s}=\tan ^{-1}\left(\frac{\sin \Phi_{s e p}-\Phi_{s e p}}{1-\cos \Phi_{s e p}}\right), \\
\Phi_{s e p}=\frac{2 \pi \hat{b}}{\beta \lambda}
\end{gathered}
$$




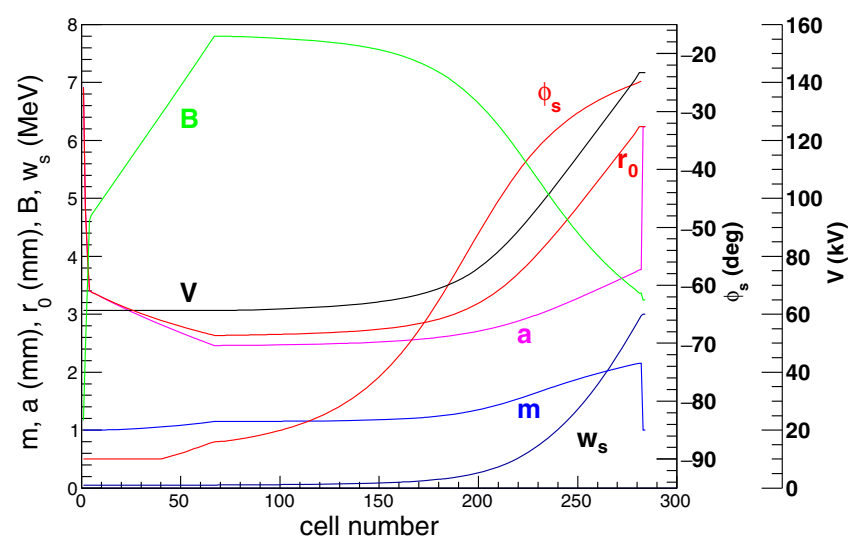

FIG. 1. Cell parameters of the fully equipartitioned RFQ. The $W_{s}$ means the energy of the synchronous particle. The $\phi_{s}$ 's after the output transition cell [13] are not plotted.

$$
\hat{b}=\left\{c_{1}+c_{2}\left(z-z_{\mathrm{EOS}}\right)\right\} b,
$$

where $\lambda$ is the $\mathrm{rf}$ wavelength, $c_{1}$ and $c_{2}$ are parameters to adjust the longitudinal acceptance, and $z_{\mathrm{EOS}}$ is $z$ at the end of SP. For the equipartitioning condition, $m$ is found by exactly solving the simultaneous equations (2), (3), and (1) for $r, b$, and $m$. The vane shape parameters are also included in obtaining the exact solution for $m$. The $\varepsilon_{t n}$ and $\varepsilon_{l n}$ are given parameters in the program.

Figure 1 shows the obtained cell parameters as functions of cell number. In Table II, the design parameters of epRFQ obtained using above scheme are summarized. The transverse cross section of the vane is a circular shape with radius $\rho_{t}$; the ratio of the $\rho_{t}$ to $r_{0}$ is constant $\left(\rho_{t} / r_{0}=0.75\right)$. The longitudinal vane profile is a two-term potential shape. The acceleration factor of a sinusoidal vane is somewhat larger than that of the two-term potential shape, and would result in an $\sim 4 \%$ shorter vane length for our case. However, the electric field distortion induced by vane-tip modulation of the sinusoidal vane is larger than that of the two-term vane. Therefore, to avoid the additional tuning difficulty, we adopted the two-term vane profile this time. Nevertheless, the obtained vane length is $15 \%$ shorter than that of RFQ III; this proves that both high acceleration efficiency and a tight, low loss beam can simultaneously be achieved with a beam oriented design.

\section{PARTICLE SIMULATION}

To evaluate the obtained design, particle simulation was performed by using LINACSrfqSIM [4,11]. LINACSrfqSIM is a time-domain code to treat the space-charge effect correctly. In addition to the conventional multipole-expansion method (mpole mode), a multigrid Poisson solver is implemented (Poisson mode). In the Poisson mode, the Poisson equation is solved with the boundary condition from the actual vane shapes to obtain both the external and space-charge fields. The Poisson solver calculates the electromagnetic field more accurately than the mpole method, especially, in the vicinity of the vane tips, and automatically correctly includes the image-charge effect. These features are important for simulating high intensity RFQs because the more particles are distributed in the vicinity of the vane tips than with low intensity beams.

In the simulation of epRFQ described in this section, the peak current of the input is $60 \mathrm{~mA}$, and the transverse distribution is water bag. The input energy is $50 \mathrm{keV}$, and the phase distribution is uniform. The normalized rms transverse emittance is $0.20 \pi \mathrm{mm} \mathrm{mrad}$ except for the transverse emittance dependence study (Fig. 6 and Table IV). In Table III, the simulation results are summarized. All of the following results are obtained using Poisson mode. The transmission is defined as a ratio of number of the accelerated to input particles.

Figure 2 shows emittance evolution as functions of the cell number. The solid line represents the transverse emittance and the dashed line is longitudinal one. The emittance growths in both directions are well controlled

TABLE II. Design parameters of epRFQ obtained with LINACSrfqDES. As a reference, the parameters of RFQ III [6] are also shown.

\begin{tabular}{lcc}
\hline \hline & epRFQ & RFQ III [6] \\
\hline Design beam current $(\mathrm{mA})$ & & 70 \\
Design transverse emittance $(\pi \mathrm{mm}$ mrad, normalized, rms) & 0.2 & 0.3 \\
Design longitudinal emittance $(\pi \mathrm{mm}$ mrad, normalized, rms) & & 3623 \\
Vane length $(\mathrm{mm})$ & 3073 & 319 \\
Number of cells & 285 & 81.0 \\
Inter-vane voltage $(\mathrm{kV})$ & $61.3-143$ & $30.7\left(1.72 E_{k}\right)$ \\
Maximum surface field $(\mathrm{MV} / \mathrm{m})$ & $30.3\left(1.70 E_{k}\right)$ & 3.49 \\
Average bore radius $\left(r_{0}\right)(\mathrm{mm}$, excluding RMS) & $2.64-6.24$ & 0.75 \\
$\rho_{t} / r_{0}$ ratio & & 2.23 \\
$a_{\min }(\mathrm{mm})$ & 2.46 & 2.13 \\
$m_{\max }(\mathrm{deg})$ & -24.8 & -30.6 \\
$\phi_{s, \text { max }}$ & 99.1 & 98.5 \\
Simulated transmission $(\%)$ & & \\
\hline \hline
\end{tabular}


TABLE III. Simulation results of epRFQ.

Input beam current Input transverse distribution Input longitudinal distribution Transmission

Output transverse emittance Output longitudinal emittance

within the acceptable level. The longitudinal phase-space distribution at the RFQ exit is shown in Fig. 3. The particles are bunched very well and no particles are distributed away from the ensemble.

Next, the parameters concerning the equipartitioning are presented in Fig. 4. It can be seen that $\varepsilon_{l n} \sigma_{l} / \varepsilon_{t n} \sigma_{t}=1$ after the exit of the SP, as the design aimed. The emittance ratio (dashed line) is around 1.3 after the exit of the SP.

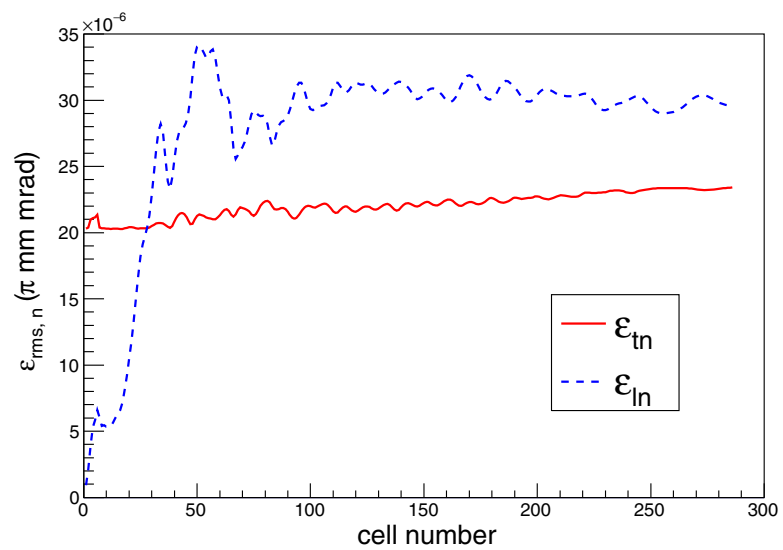

FIG. 2. Emittance evolution as functions of the cell number. The solid line represents the transverse normalized rms emittance, and the dashed line is the longitudinal one.

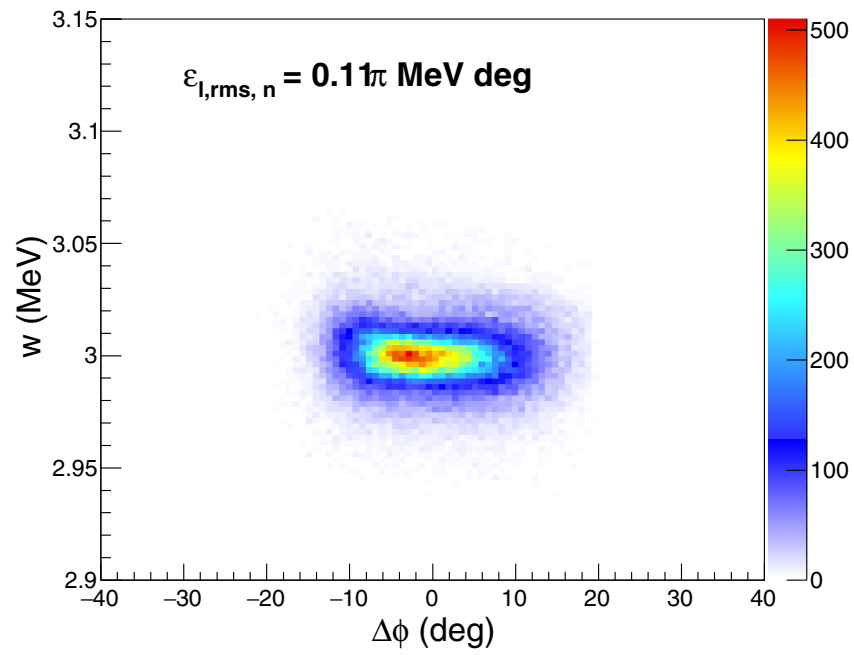

FIG. 3. Simulated longitudinal particle distribution at the exit of the RFQ.

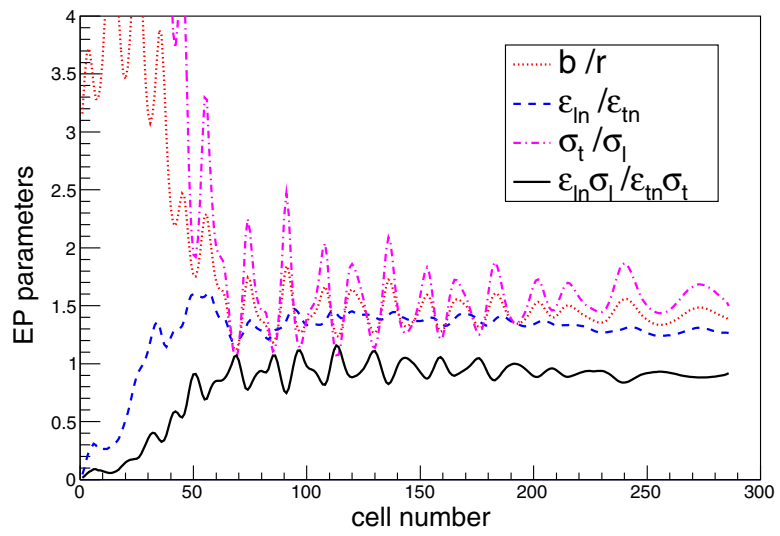

FIG. 4. Parameters related to the equipartitioning condition (EP parameters). When the equipartitioning condition holds, the solid line should be one.

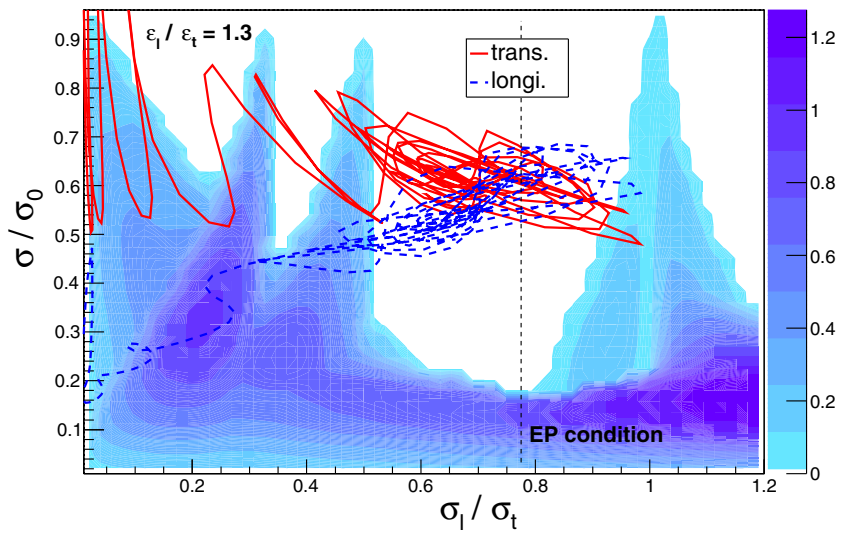

FIG. 5. Trajectories of the phase-advance ratio and depression of epRFQ on the Hofmann chart. The longitudinal/transverse emittances ratio of this Hofmann chart is 1.3.

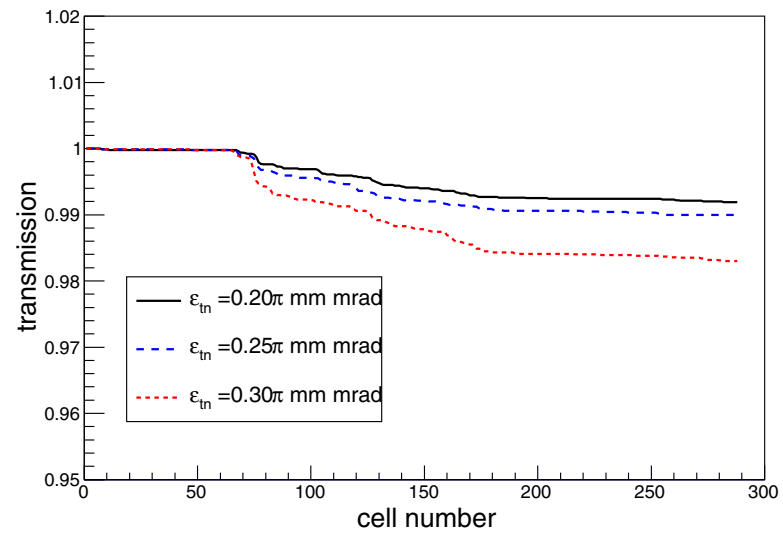

FIG. 6. Input-transverse-emittance dependence of the transmission. The solid, dashed, and dotted lines are transmissions for the input transverse emittances of $0.20,0.25$, and $0.30 \pi \mathrm{mm} \mathrm{mrad}$, respectively. 
TABLE IV. Input-transverse-emittance dependence of the epRFQ simulation.

\begin{tabular}{llll}
\hline \hline Input emittance $(\pi \mathrm{mm}$ mrad$)$ & 0.20 & 0.25 & 0.30 \\
Transmission $(\%)$ & 99.1 & 98.9 & 98.2 \\
Output trans. emittance $(\pi \mathrm{mm}$ mrad) & 0.24 & 0.28 & 0.32 \\
Output longi. emittance $(\pi \mathrm{MeV} \mathrm{deg})$ & 0.11 & 0.11 & 0.12 \\
\hline \hline
\end{tabular}

Figure 5 shows a Hofmann chart [14] for the emittance ratio of 1.3; the chart is specific to the emittance ratio. The horizontal axis of the Hofmann chart represents the ratio of the longitudinal phase advance to the transverse one (tune ratio), and the vertical axis is the ratio of the phase advance with space charge to that without space charge (tune depression). By plotting the trajectory of the tune depression as a function of the tune ratio on this plot, the status of equipartitioning achievement and resonance crossing can be checked. In Fig. 5, the solid and dashed lines represent the transverse and longitudinal trajectories of epRFQ in this space, respectively. The line "EP condition" shows where Eq. (1) holds. After the exit of the SP, the trajectories are around the EP condition, where there is no energy to drive resonances, therefore emittance growth is small.

Finally, the dependence of the transmission on the input emittance is shown in Fig. 6 and the transmissions and emittances are summarized in Table IV. The solid, dashed, and dotted lines represent transmissions for the input transverse emittances of $0.20,0.25$, and $0.30 \pi \mathrm{mm} \mathrm{mrad}$, respectively. After the EOS (cell 67), the bunching is adiabatic, but before that, it is not; this causes the slight beam loss. If the $\phi_{s}$ at the EOS ( $-87^{\circ}$ for epRFQ and $-86^{\circ}$ for RFQ III) is closer to $-90^{\circ}$, this loss may be decreased, but results in longer RFQ. At present, the transverse emittance of the J-PARC ion source is typically $0.27-0.30 \pi \mathrm{mm} \mathrm{mrad}$, therefore, the output emittance of around $0.3 \pi \mathrm{mm}$ mrad is expected at the RFQ exit with a sufficient transmission; this is acceptable enough for the following linac [15].

\section{BEAM COMMISSIONING}

\section{A. Experimental apparatus}

The epRFQ was beam commissioned at the RFQ test stand [5] on the ground floor of the J-PARC linac building. The RF driven ion source and the low energy beam transport (LEBT) are basically same as the previous experiment.

Figure 7 shows a photograph of epRFQ installed in the test stand. The basic cavity structure of epRFQ is same as that of RFQ III. Two 1700-L/s (for $\mathrm{N}_{2}$ ) cryo pumps are attached to the upstream part. Three vacuum ports connected with a manifold are used for each cryo pump. Two $400-\mathrm{L} / \mathrm{s}$ ion pumps are attached to the downstream part of the RFQ. Typical pressure was $5 \times 10^{-5} \mathrm{~Pa}$ under $50-\mathrm{mA}$ beam operation. In the center port of the lower-right

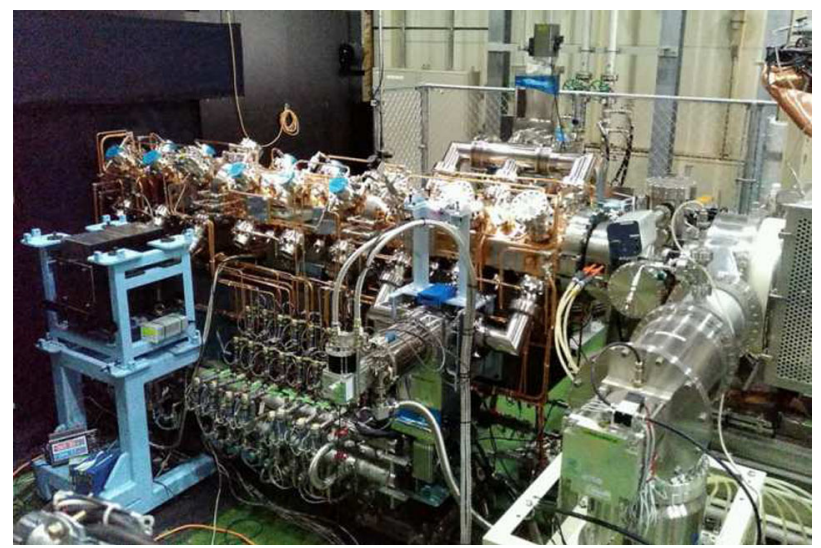

FIG. 7. Photograph of epRFQ installed in the test stand.

quadrant, a loop-type if coupler is inserted. The rf power was generated by a 324-MHz klystron (Canon ETD E3740A [16]).

The property of the extracted beam from the RFQ was measured using a test bench. Figure 8 is a schematic side view of the test bench. The test bench is installed in the black hut shown at the downstream of the RFQ in Fig. 7. The beam from the RFQ was transported to the beam dump using three quadrupole magnets $(\mathrm{Q} 1, \mathrm{Q} 2$, and Q3). The beam current was measured with a slow current transformer (SCT), and the beam phase was measured with a fast current transformer (FCT) [17,18]. The emittance monitor is a conventional double-slit type to measure the transverse emittances of the RFQ. The slit of the emittance monitor is composed of tungsten, which is water cooled via a copper baking plate. The gap length of the slit is $0.1 \mathrm{~mm}$, and the distance between the upstream slit and the downstream Faraday cup with a slit is $215 \mathrm{~mm}$. The charges of the particles passing through the two slits are collected by the Faraday cup. The slit and Faraday cup are driven by stepping motors. The same data acquisition system of the emittance monitors used in the RFQ III commissioning was utilized. Finally, the beam was directed to the beam dump. The beam dump consists of a copper cylinder cut with an angle of 30 degrees and a 1-mm tungsten plate attached to the cut surface of the cylinder by the hot

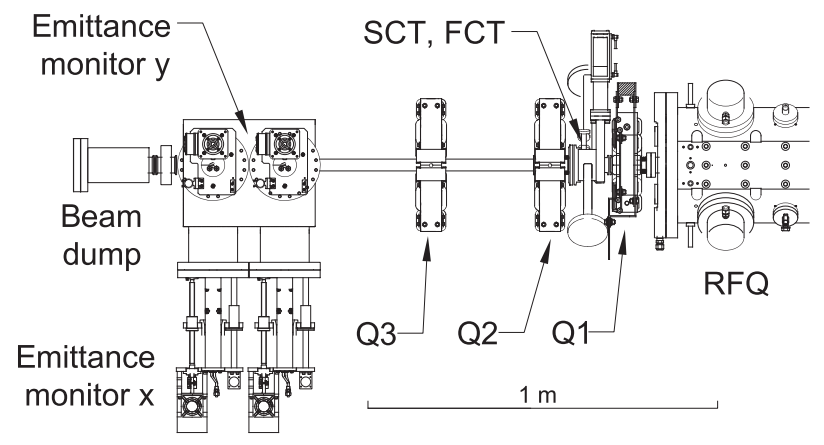

FIG. 8. Schematic side view of the test bench. 

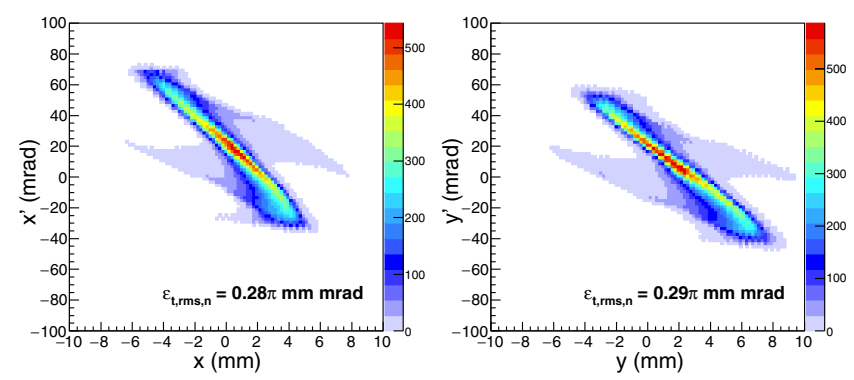

FIG. 9. Phase space distribution of the ion source measured just after the first solenoid magnet of the ion source test stand. The measured beam current was $60 \mathrm{~mA}$.

isotropic pressing method. Because contamination by impurities such as nickel and copper cause neutron radiation, a high purity tungsten was used for the beam target.

\section{B. Beam commissioning results}

In this subsection, the beam-commissioning result of epRFQ is presented and compared with simulation.

For the input distribution into the RFQ simulation, the generated distribution based on the measurement at the LEBT was used. Figure 9 shows the measured phase space distribution at the LEBT. Because the LEBT of the RFQ test stand is not equipped with emittance monitors, the distribution of the ion source had been measured at another ion source test stand prior to installation into the RFQ test stand. It is assumed that there is no significant difference between the effects of the space charge neutralization of the two test stands. The input distribution for LINACSrfqSIM was reconstructed from the measured distribution using genBeam [19].

Figure 10 shows the inter-vane voltage dependence of the transmission through the RFQ. The $V_{n}$ denotes the

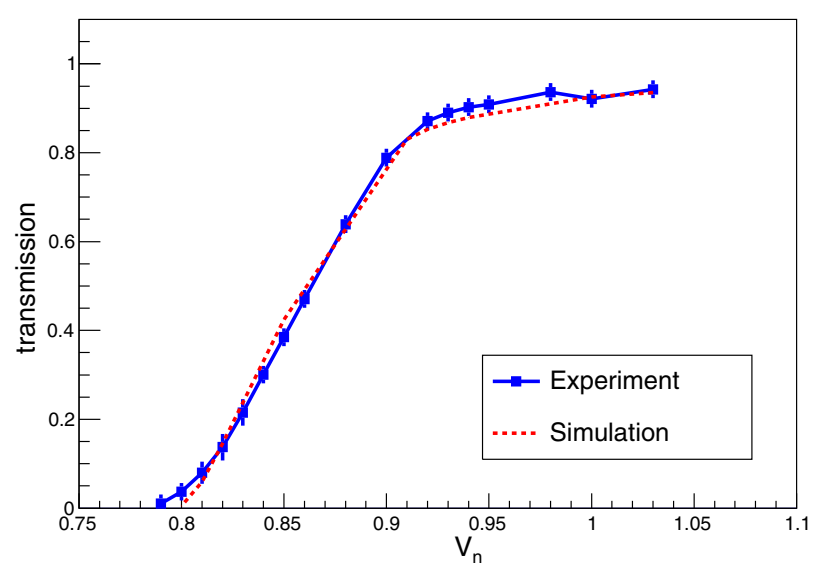

FIG. 10. Measured and simulated transmissions of epRFQ as functions of the inter-vane voltage $V_{n}$. This measurement was performed with a beam width of $50 \mu \mathrm{s}$ and a repetition rate of $25 \mathrm{~Hz}$. The error bars are derived from the fluctuation of the spectrum measurement using the FCT.
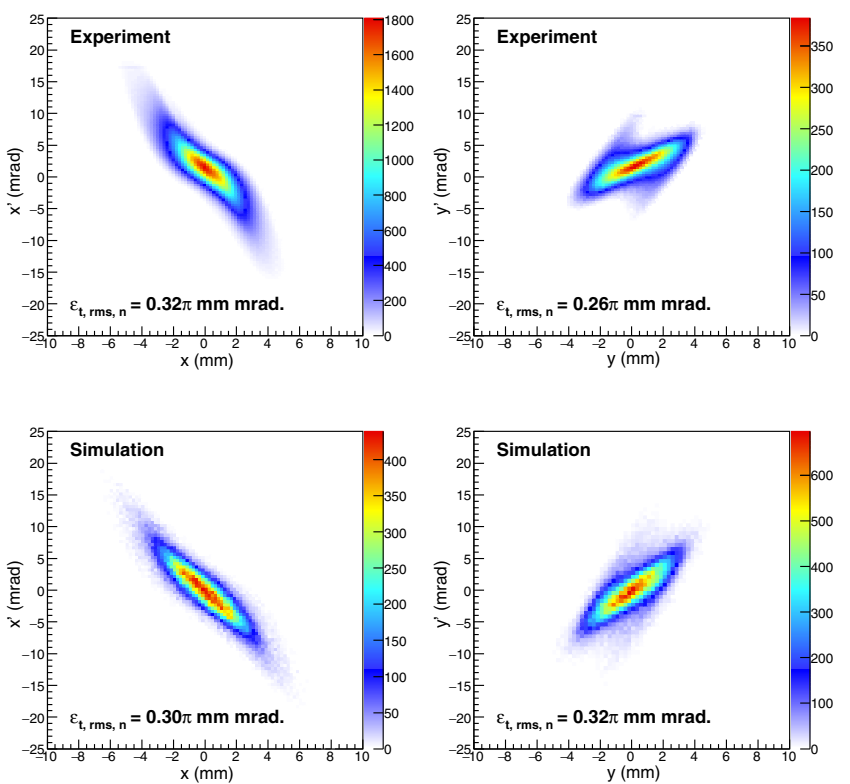

FIG. 11. Measured (upper row) and simulated (lower row) transverse emittances of epRFQ.

inter-vane voltage of the RFQ normalized to the design voltage, and the vertical axis is the transmission. The solid and dashed lines represent the measured and simulated transmissions of the accelerated particles, respectively. The accelerated beam current was obtained by measuring the 324-MHz spectrum of the FCT signal using a Tektronix [20] MDO4014B-3 mixed-domain oscilloscope. The absolute beam current at the RFQ exit was measured using the SCT of the test bench. The measured beam current was normalized by the RFQ injection current of $59 \mathrm{~mA}$ measured with the SCT of the LEBT. The extracted current from the RFQ at the design voltage was $54 \mathrm{~mA}$, thus the transmission was $92 \%$, which agrees with the simulated transmission of $93 \%$.

Next, the results of the transverse emittance measurements of the extracted 54-mA beam are shown in Fig. 11. To prevent melting of the edge of the slits, the emittances of the 54-mA beam were measured with a width of $50 \mu \mathrm{s}$ and a repetition rate of $25 \mathrm{~Hz}$. PARMILA [21] was employed for the test bench simulation. In Fig. 11, the upper row shows the measured transverse emittances and the lower row gives the simulation results. The measured values of the normalized rms transverse emittances $\varepsilon_{t, \mathrm{rms}, n}$ in the horizontal and vertical planes were $0.32 \pi \mathrm{mm} \mathrm{mrad}$ and $0.26 \pi \mathrm{mm} \mathrm{mrad}$, whereas the simulated emittances were $0.30 \pi \mathrm{mm} \mathrm{mrad}$ and $0.32 \pi \mathrm{mm} \mathrm{mrad}$, respectively. Possible uncertainty of the emittance measurement is as follows. The rms emittance value strongly depend on the halo like particles, and the rms emittance becomes small if these particles are scraped. In the simulation, RFQ injection is radially symmetric, however in reality, there are an electron kicker and an angle correction magnet in the $y$ direction in the ion source. Therefore, there is a 
possibility the beam is scraped in only the y direction through the RFQ. Note that this affects not strong impact to the transmission curve because the scraped current itself is small. The second is that the ion source emittances at the RFQ test stand are different from those measured at ion source test stand. The emittance of the ion source may fluctuate a few tens $\%$ even with same setup and setting. The other is systematics of the emittance monitor itself. Taking account of this uncertainty, the simulation well reproduces the measurements. It is important to note that the beam also remains "tight," retaining its total emittance characteristics as well as expected for equipartitioned transport.

From above results, the performance of epRFQ conforms to its design.

\section{CONCLUSION}

We have developed a fully equipartitioned RFQ (eqRFQ), which accelerates a negative hydrogen beam from $50 \mathrm{keV}$ to $3 \mathrm{MeV}$. In the design of this RFQ, flexible rules for $V, a, \phi_{s}$ are adopted, and $m$ is used to solve for the correct space-charge physics: that is, the equipartitioning design scheme, which is a defacto standard in the high intensity linac design, is implemented. For the beam-dynamics design, LINACSrfqDES was used and LINACSrfqSIM was employed for the particle simulation. Under the condition of input beam-current of $60 \mathrm{~mA}$ and transverse emittance of $0.20 \pi \mathrm{mm}$ mrad, the transmission of $99.1 \%$, output transverse emittance of $0.24 \pi \mathrm{mm} \mathrm{mrad}$, and longitudinal emittance of $0.11 \pi \mathrm{MeV}$ deg are obtained.

A beam test of epRFQ was successfully conducted, and the experimental data were compared with the simulation results. Operational stability was same as that of RFQ III at the test stand, thus this epRFQ satisfies the requirements as a spare of the J-PARC RFQ. A realistic distribution based on the measured distribution at the LEBT was used for the input distribution of the RFQ simulation. The measured transmission of the $59 \mathrm{~mA}$ input beam was $92 \%$, whereas the simulated transmission was $93 \%$. The horizontal and vertical emittances were measured to be $0.32 \pi \mathrm{mm} \mathrm{mrad}$ and $0.26 \pi \mathrm{mmmrad}$, and the simulation results were $0.30 \pi \mathrm{mm} \mathrm{mrad}$ and $0.32 \pi \mathrm{mmmrad}$, respectively. It is very important to note the very close agreement between the experimental results and the simulation, because this verifies the complete progression from design to experimental result of a beam physics based method.

From these results, it is concluded that the RFQ which accomplished the aim of the equipartitioning design concept are realized.

\section{ACKNOWLEDGMENTS}

The authors would express appreciation to Professor I. Hofmann for providing the data of Hofmann chart. We express our appreciation to Mitsubishi Heavy Industries
Machinery Systems Ltd. for their remarkable contribution to the fabrication of epRFQ.

[1] K. R. Crandall, R. H. Stokes, and T. P. Wangler, in Proceedings of 1979 Linear Accelerator Conference (Montauk, New York, USA, 1979), pp. 205-216.

[2] L. M. Young, in Proceedings of the Particle Accelerator Conference, Vancouver, BC, Canada, 1997 (IEEE, New York, 1997), pp. 2752-2754.

[3] M. Comunian, A. Pisent, E. Fagotti, and P. A. Posocco, in Proceedings of the 11th European Particle Accelerator Conference, Genoa, 2008 (EPS-AG, Genoa, Italy, 2008), pp. 3536-3538.

[4] R. A. Jameson, RFQ designs and beam-loss distributions for IFMIF, Oak Ridge National Laboratory Technical Report No. ORNL/TM-2007/001, 2007.

[5] Y. Kondo et al., Beam test of a new radio frequency quadrupole linac for the Japan Proton Accelerator Research Complex, Phys. Rev. Accel. Beams 17, 120101 (2014).

[6] Y. Kondo, K. Hasegawa, T. Morishita, and R. A. Jameson, Beam dynamics design of a new radio frequency quadrupole for beam-current upgrade of the Japan Proton Accelerator Research Complex linac, Phys. Rev. Accel. Beams 15, 080101 (2012).

[7] R. A. Jameson, Beam-intensity limitations in linear accelerators, IEEE Trans. Nucl. Sci. 28, 2408 (1981).

[8] Equations (2)-(5) describe generalized situation not depend on the particular structure and resonant frequency. Therefore, the unit length $L$ is more suitable than the particular wavelength, so we changed the notation from [6].

[9] T. P. Wangler, Space-charge limits in linear accelerators, Los Alamos National Laboratory Technical Report No. LA-8388, 1980.

[10] K. R. Crandall, Effects of vane-tip geometry on the electric fields in radio-frequency quadrupole linacs, Los Alamos National Laboratory Technical Report No. LA-9695-MS, 1983.

[11] R. A. Jameson, LINACS design and simulation framework, KEK/J-PARC Technical Report No. KEK/J-PARC Seminar 6 March 2012.

[12] W. D. Kilpatrick, Criterion for vacuum sparking designed to include both rf and dc, Rev. Sci. Instrum. 28, 824 (1957).

[13] K. R. Crandall, in Proceedings of the 17th International Linear Accelerator Conference (LINAC-1994), Tsukuba, Japan, 1994 (KEK, Tsukuba, Japan, 1994), pp. 227-229.

[14] I. Hofmann, G. Franchetti, O. Boine-Frankenheim, J. Qiang, and R. D. Ryne, Space charge resonances in two and three dimensional anisotropic beams, Phys. Rev. Accel. Beams 6, 024202 (2003).

[15] With more complex beam-based design techniques (beyond the scope of this project), even more constant emittances, more exact EP parameters along the trajectories and lower losses would be expected.

[16] Canon Electron Tubes and Devices Co. Ltd., https://etd .canon/.

[17] S. Sato, T. Tomisawa, Y. Kondo, A. Ueno, H. Akikawa, Z. Igarashi, S. Lee, C. Kubota, and M. Ikegami, in 
Proceedings of the 23rd International Linac Conference, LINAC-2006, Knoxville, TN, 2006 (JACoW, Knoxville, TN, 2006), pp. 268-270.

[18] A. Miura, T. Miyao, and Z. Igarashi, in Proceedings of LINAC2012 (Tel-Aviv, Israel, 2012) pp. 699-701.
[19] D. Bouma and J. Stovall, genBeam, CERN Technical Report, 2009.

[20] Tektronix Inc., http://www.tek.com/.

[21] H. Takeda, Parmila, Los Alamos National Laboratory Technical Report No. LA-UR-98-4478. 